OPEN ACCESS

Edited by:

Zhikuan Yang,

Central South University, China

Reviewed by:

Zhong-Lin Lu,

New York University, United States

Anna Horwood,

University of Reading,

United Kingdom

*Correspondence:

Xinping Yu

yu-xinping@163.com

Specialty section: This article was submitted to

Perception Science,

a section of the journal

Frontiers in Neuroscience

Received: 25 March 2021 Accepted: 02 June 2021

Published: 05 July 2021

Citation:

Peng T, Xu M, Zheng F, Zhang J, Chen S, Lou J, Wang C, Wang Y and

YuX (2021) Longitudinal Rehabilitation of Binocular Function in Adolescent Intermittent Exotropia After Successful Corrective Surgery.

Front. Neurosci. 15:685376

doi: 10.3389/fnins.2021.685376

\section{Longitudinal Rehabilitation of Binocular Function in Adolescent Intermittent Exotropia After Successful Corrective Surgery}

\author{
Tingting Peng', Meiping X ${ }^{1}$, Fuhao Zheng ${ }^{1}$, Junxiao Zhang ${ }^{1}$, Shuang Chen ${ }^{1}$, \\ Jiangtao Lou' ${ }^{1}$, Chunxiao Wang ${ }^{1}$, Yuwen Wang ${ }^{1}$ and Xinping Yu ${ }^{1,2 *}$ \\ ${ }^{1}$ The Eye Hospital, School of Ophthalmology and Optometry, Wenzhou Medical University, Wenzhou, China, ${ }^{2}$ State Key
Laboratory of Ophthalmology, Zhongshan Ophthalmic Center, Sun Yat-sen University, Guangzhou, China
}

Purpose: To study the longitudinal rehabilitation of binocular visual function in adolescent intermittent exotropia (IXT) after successful surgery and compare the results with those of a normal population. The role of binocular function in ocular alignment stability was also evaluated postoperatively.

Methods: In this prospective study, 30 adolescents with IXT successfully corrected after 1 month were followed for 12 months, and 30 children with normal vision were enrolled as controls. Stereopsis, the fusional vergence amplitude, sensory fusion, and accommodative flexibility were measured to assess binocular function at baseline and 6 and 12 months postoperatively. The controls were tested once when they were enrolled in the study.

Results: The deviation was $-32.00 \pm 8.60$ prism diopters $(P D)$ at distance fixation and $-36.0 \pm 9.10 \mathrm{PD}$ at near fixation preoperatively with an average correction of $28.53 \pm 3.79 \mathrm{PD}$ and $30.67 \pm 1.34 \mathrm{PD}$ at 1 month postoperatively. Distance stereoacuity and near stereoacuity improved from 1 to 12 months postoperatively ( $p=0.025$ and $p=0.041$, respectively). Compared with the controls, the fusional convergence reserve at distance $(p=0.025)$ and near $(p=0.033)$ fixations and fusion reserve ratio at distance $(p=0.000)$ and near $(p=0.000)$ fixations remained subnormal, whereas sensory fusion $(p=0.237)$, distance stereopsis $(p=0.120)$, and the fusional divergence amplitude at a distance $(p=0.168)$ were normal. However, no significant correlations were found between binocular functions at 1 month postoperatively and the postoperative drift.

Conclusion: Binocular function significantly improved from before to after successful corrective surgery and continued to improve from 1 to 12 months postoperatively in adolescents with IXT. No significant correlations were found between binocular functions at 1 month postoperatively and ocular alignment stability.

Keywords: intermittent exotropia, strabismus surgery, binocular function, rehabilitation, ocular alignment 


\section{INTRODUCTION}

Intermittent exotropia (IXT) is the most common form of exotropia in children and adolescents. The prevalence of IXT is approximately 1\% in Western countries (Hutchinson, 2001) and approximately $3 \%$ in teenagers in China (Pan et al., 2016). IXT is characterized by intermittent fusion when the eyes experience proper ocular alignment in the early stage of strabismus, leading to the development of binocularity and stereopsis; patients with IXT possess the potential to regain normal binocular functions after treatment (Donahue, 2007). Surgical treatment is critical for IXT (Jie et al., 2010; Yu et al., 2016) in children and adolescents. Binocular functions (e.g., stereopsis, sensory fusion, and motor fusion) improve after surgery (O’Neal et al., 1995; Yildirim et al., 1999; Wu et al., 2006; Adams et al., 2008; Sharma et al., 2008; Feng X. et al., 2015; Pineles et al., 2015; Wu Y. et al., 2020). Feng X. et al. (2015) found that stereopsis and sensory fusion improved from 2 to 6 weeks postoperatively, whereas binocular fusion and alignment were not evaluated over longer periods. Distance stereoacuity improved in individuals with IXT at 6 weeks (Adams et al., 2008) and 1 year after successful surgery (Yildirim et al., 1999; Wu et al., 2006). Sharma et al. (2008) found an increase in fusional vergence and both near and distance stereoacuities 6 months after surgery in individuals with IXT. However, these studies performed only one follow-up after surgery. Longitudinal rehabilitation in binocular function after successful surgery as well as their associations with ocular alignment drift were not evaluated. The longitudinal recovery of binocular function in IXT patients after successful surgery should be evaluated extensively.

The high recurrence rate after surgery reduces the benefits of corrective surgery in IXT patients. The specific factors for recurrence or drift after surgery in IXT have not yet been established. However, factors, such as the surgical procedure, age at surgery, angle of deviation, and preoperative binocular functions, have been studied previously (Pineles et al., 2010; Lee et al., 2018; Mohan and Sharma, 2020; Repka et al., 2020). Postoperative binocular function was not related to postoperative ocular alignment stability in our retrospective study (Wu Y. et al., 2020). An adequate understanding of the recovery of binocular function after successful surgery may help identify the factors associated with the recurrence of IXT after surgery.

Based on these findings, we designed a prospective study to evaluate the longitudinal rehabilitation of binocular visual function (e.g., sensory fusion, stereoacuity, fusional vergence amplitude, amplitude of accommodation, accommodative flexibility) after successful surgery to compare the results with those of a normal population and to determine whether binocular function is involved in the stability of ocular alignment postoperatively.

\section{SUBJECTS AND METHODS}

This prospective case-control study was approved by the Ethics Committee of the Eye Hospital of Wenzhou Medical University and was performed in accordance with the Declaration of Helsinki.

\section{Subjects}

Thirty patients aged 7-17 years with IXT successfully corrected for 1 month after surgery and 30 asymptomatic age- and sexmatched controls were recruited from the Eye Hospital of Wenzhou Medical University from July 2019 to September 2019. Only patients with basic IXT (Donahue et al., 2019) preoperatively were included in the study. The definition of successful motor alignment is orthotropia, $\mathrm{X}(\mathrm{T}) \leq 10$ prism diopters $(\mathrm{PD})$ and $\mathrm{E}(\mathrm{T}) \leq 5 \mathrm{PD}$ in the primary position at distance and near fixations (Kim and Choi, 2016; Donahue et al., 2019). Patients were excluded if any of the following conditions were encountered: diplopia at 1 month after surgery, vertical deviation $\geq 5 \mathrm{PD}$, dissociated vertical deviation (DVD), oblique muscle dysfunction, an $\mathrm{A}$ or $\mathrm{V}$ pattern, eye movement restricted in one direction, a congenital cranial nerve abnormality, a history of extraocular muscle surgery or botulinum toxin injection treatment, amblyopia ( $\geq 2$ lines interocular difference on Snellen's vision chart), anisometropia (a spherical or cylindrical difference $\geq 2.0$ diopters), or a history of binocular vision therapy pre- or postoperatively.

\section{Data Collection}

The following data were recorded for each patient: name, sex, age, age at the time of surgery, best-corrected visual acuity (BCVA), eye movements (EOM), preoperative and postoperative angle of deviation and binocular functions, including sensory (sensory fusion, stereoacuity) and motor function (fusional vergency amplitude, amplitude of accommodation, and accommodative flexibility).

The angle of deviation was measured using the prism and alternate cover test (PACT) at $33 \mathrm{~cm}$ for near and $6 \mathrm{~m}$ for distance fixations. The deviation of tropia was measured using the simultaneous prism and cover test (SPCT) at $33 \mathrm{~cm}$ for near fixation and $6 \mathrm{~m}$ for distance fixation (Donahue et al., 2019).

Sensory fusion was tested using the Worth 4-dot test at distance and near fixations. Patients who detected four dots were considered to have fusion, those who saw five dots were considered diplopic, and those who saw two or three dots were considered to have suppression. Subjects who saw 2 or 3 dots at distance fixation and 4 dots at near fixation were defined as having peripheral fusion (Yildirim et al., 1999; Feng X. et al., 2015).

Near stereoacuity was assessed using TNO stereopsis tests at $40 \mathrm{~cm}$ (Laméris Ootech B.V., Nieuwegein, Netherlands), ranging from 15 to $480 \mathrm{~s}$ of $\operatorname{arc}(\operatorname{arcsec})$ (Wu Y. et al., 2020). Distance stereoacuity was assessed using the Distance Randot Stereotest (DRS, American Stereo Optical Company) at $3 \mathrm{~m}$, ranging from 63 to 400 arcsec (Wang et al., 2010). Stereoacuity was recorded as "nil" if the patient could not pass the largest disparity (Hatt et al., 2008). The stereopsis examination was performed before any other examination that required binocular fusion to be broken.

The fusional vergence amplitude was measured using a horizontal prism bar, and an accommodative target was used first at distance fixation $(3 \mathrm{~m})$ and then at near fixation $(1 / 3 \mathrm{~m})$ (Sharma et al., 2008; Hatt et al., 2011) with basein (BI) for negative vergence and base-out (BO) for positive 
vergence. Negative fusional vergence was measured before positive vergence to prevent bias caused by the prismatic demand of positive fusional vergence. To determine the fusional convergence break point (convergence reserve), the magnitude of prism was gradually increased from 1 PD until diplopia appeared with no subsequent recovery of motor fusion or one eye drifted outwards when control was lost. If the patient could still perform fusion up to the maximum prism volume of $45^{\Delta}$, the break point was recorded as $45^{\Delta}$ for statistical analysis (Hatt et al., 2011). If a patient could not perform fusion, both the break point and recovery point were recorded as 0 . The total fusional convergence amplitude (Hatt et al., 2011) was the sum of the individual deviation angle and convergence reserve. The fusion reserve ratio (Hatt et al., 2011) was calculated as the fusional convergence reserve divided by the angle of deviation measured using the PACT (e.g., fusional convergence reserve $=20$; angle of deviation $=10$; fusion reserve ratio $=2$ ).

For the near point of convergence (NPC), the patient looked at an accommodative target located $40 \mathrm{~cm}$ away, and the examiner gradually moved the target toward the patient's eyes until the patient reported that the target had become two targets. The distance between the break point and parallel point of the patient's lateral canthus was measured.

The amplitude of accommodation (AMP) was measured using the negative lens method, and the right eye's data were used for analysis.

Binocular accommodative flexibility (BAF) was measured by reading the "E" visual acuity chart at $40 \mathrm{~cm}$ in sequence with $\mathrm{a} \pm 2.00 \mathrm{D}$ reverse lens within $1 \mathrm{~min}$ (García et al., 2000; Wick et al., 2002). Positive and negative counts comprise one cycle. The measurement starts with the positive lens, and the number of cycles that occur within $1 \mathrm{~min}$ is recorded.

All the tests were performed after appropriate refractive correction. Each of these tests was performed at 1, 6, and 12 months postoperatively. Two patients were lost to follow-up at 6 months after surgery. The controls were tested once when they were enrolled in the study.

\section{Statistical Analysis}

Postoperative drift was defined as the change in ocular alignment from the 1-month follow-up to the final follow-up. The Friedman test was used to compare the angle of deviation, stereoacuity, and fusional vergence amplitude (1, 6, and 12 months). The Wilcoxon signed-rank test was used to compare rehabilitation between 1 and 12 months postoperatively, and the MannWhitney U test was used to compare IXT patients and controls. The sensory fusion status at each time point (1, 6, and 12 months) was evaluated using chi-squared test or Fisher's exact test. Additionally, we evaluated the relationships between postoperative binocular functions and postoperative drift using Spearman's correlation coefficient.

\section{RESULTS}

Thirty cases and 30 age- and sex-matched controls were included. A summary of the subjects' demographics and clinical characteristics is provided in Table 1. The deviations at both distance and near fixations decreased significantly from preoperatively to 1 month postoperatively ( $p<0.001)$, sensory fusion improved ( $p=0.009)$, and distance stereoacuity improved ( $p=0.026)$; however, near stereoacuity did not recover significantly $(p=0.657)$.

\section{Postoperative Drift}

Figure 1A shows that the eye position steadily drifted outwards. The mean exodrift at distance fixation was $1.53 \pm 7.19 \mathrm{PD}$ from postoperative month 1 to postoperative month 6 and $2.67 \pm 9.38$ PD from postoperative month 6 to postoperative month 12 $(p=0.338)$, and the mean exodrift values at near fixation were $2.00 \pm 9.83 \mathrm{PD}$ and $2.97 \pm 11.62 \mathrm{PD}$, respectively $(p=0.459)$. No relationship was found between the preoperative deviation and postoperative drift at distance or near fixations $[r s=0.352$ $(p=0.056)$ and $r s=0.048(p=0.802)$, respectively]. The greater was the amount of preoperative deviation, the greater was the postoperative drift at a distance. However, the difference was not statistically significant.

\section{Binocular Functions After Surgery Sensory Fusion}

No significant differences were found in sensory fusion between 1 and 12 months postoperatively $(p=0.601)$ or between the patient and control groups ( $p=0.117$ and $p=0.237$, respectively).

\section{Stereoacuity}

The patients' median value of distance stereoacuity improved from 3.18 (range, 1.8-4.0) at 1 month postoperatively to 2.77 (range, $1.8-4)$ at 12 months postoperatively $(p=0.025)$, and the values did not significantly differ from those of the controls $(p=0.120)$. The patients median value of near stereoacuity improved from 2.20 (range, 1.7-2.98) at 1 month postoperatively to 2.03 (range, 1.48-2.68) at 12 months postoperatively $(p=0.041)$, which was still poorer than that of the controls $(p=0.017)$. Distance stereoacuity before and after surgery ( 1 month and 12 months postoperatively) did not significantly differ $[r s=0.308(p=0.098)$ and $r s=0.210$ $(p=0.266)$, respectively], whereas near stereoacuity was significantly different $[r s=0.492(p=0.006)$ and $r s=0.547$ $(p=0.002)$, respectively]. These results demonstrated that the better was preoperative near stereoacuity, the better was the postoperative recovery.

\section{Fusional Vergence Amplitude}

The patients' median fusional vergence amplitudes, total fusional convergence amplitudes, and fusion reserve ratio at distance or near fixation improved at the three follow-ups (Figure 1B-E). However, the number of distance and near BO break points in the IXT group at the final visit was still worse than that in the controls ( $p=0.025$ and $p=0.033$, respectively). The number of BI break points in the IXT group at the final visit at distance fixation was similar to that of the controls, whereas the number at near fixation exceeded that of the controls $(p=0.168$ and $p=0.003$, respectively). The distance and near-total fusional convergence amplitudes in the IXT group were similar to those of the 
TABLE 1 | Demographics and clinical characteristics of the patients with IXT and normal controls.

\begin{tabular}{|c|c|c|c|c|c|}
\hline \multirow[t]{2}{*}{ Characteristic } & \multicolumn{4}{|c|}{ IXT patients } & \multirow[t]{2}{*}{ Normal controls } \\
\hline & Preoperatively & $\begin{array}{c}1 \text { month } \\
\text { postoperatively }\end{array}$ & $\begin{array}{c}6 \text { months } \\
\text { postoperatively }\end{array}$ & $\begin{array}{c}12 \text { months } \\
\text { postoperatively }\end{array}$ & \\
\hline Sex: female, male & - & 11,19 & 11,17 & 11,19 & 10,20 \\
\hline Age (years) & - & $10.87 \pm 2.40$ & - & - & $9.90 \pm 2.06$ \\
\hline SE of the right eye (D) & - & $-2.20 \pm 2.38$ & $-2.11 \pm 2.13$ & $-2.48 \pm 2.37$ & $-0.97 \pm 1.33$ \\
\hline SE of the left eye (D) & - & $-2.00 \pm 2.39$ & $-1.67 \pm 2.05$ & $-1.90 \pm 2.77$ & $-0.87 \pm 1.20$ \\
\hline $\begin{array}{l}\text { Sensory fusion (fusion, peripheral } \\
\text { fusion, no fusion) }\end{array}$ & $15,9,6$ & $26,3,1$ & $27,1,0$ & $27,3,0$ & $30,0,0$ \\
\hline Distance stereoacuity (log arcsec) & $3.55 \pm 0.77$ & $3.18 \pm 0.97$ & $2.79 \pm 0.88$ & $2.77 \pm 0.85$ & $2.33 \pm 0.63$ \\
\hline Near stereoacuity (log arcsec) & $2.24 \pm 0.44$ & $2.20 \pm 0.34$ & $2.06 \pm 0.34$ & $2.03 \pm 0.35$ & $1.98 \pm 0.29$ \\
\hline Distance deviation (PD) & $-32.0 \pm 8.60$ & $-3.47 \pm 4.81$ & $-5.36 \pm 6.63$ & $-7.67 \pm 7.70$ & $-0.23 \pm 0.57$ \\
\hline Near deviation (PD) & $-36.0 \pm 9.10$ & $-5.33 \pm 7.76$ & $-7.86 \pm 8.29$ & $-10.30 \pm 9.87$ & $-2.27 \pm 3.04$ \\
\hline Distance base-out & - & $15.43 \pm 13.78$ & $15.50 \pm 11.89$ & $18.70 \pm 15.14$ & $23.70 \pm 9.90$ \\
\hline Distance base-in & - & $6.14 \pm 4.47$ & $7.68 \pm 5.02$ & $10.23 \pm 5.58$ & $8.67 \pm 2.37$ \\
\hline Near base-out & - & $21.43 \pm 15.39$ & $22.14 \pm 11.14$ & $24.67 \pm 14.48$ & $32.37 \pm 9.29$ \\
\hline Near base-in & - & $9.86 \pm 6.41$ & $11.89 \pm 6.93$ & $17.07 \pm 8.28$ & $11.90 \pm 3.97$ \\
\hline $\mathrm{NPC}(\mathrm{cm})$ & - & $4.00 \pm 4.21$ & $6.13 \pm 4.61$ & $4.13 \pm 4.53$ & $2.25 \pm 2.88$ \\
\hline $\begin{array}{l}\text { Binocular accommodative flexibility } \\
\text { (BAF) }\end{array}$ & - & $9.67 \pm 4.30$ & $11.64 \pm 3.97$ & $12.38 \pm 3.45$ & $9.03 \pm 3.34$ \\
\hline $\mathrm{AMP}(\mathrm{D})$ & - & $8.83 \pm 3.26$ & $8.33 \pm 2.47$ & $8.18 \pm 2.16$ & $7.37 \pm 2.16$ \\
\hline
\end{tabular}

$D$, diopters. $P D$, prism diopters.

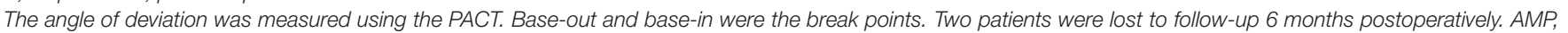
the right eye's amplitude of accommodation.

controls at the final visit ( $p=0.619$ and $p=0.935$, respectively). The fusion reserve ratio at 12 months postoperatively differed significantly between the patient and control groups at both distance $(p=0.000)$ and near $(p=0.000)$ fixation.

\section{Binocular Accommodative Flexibility}

The patients' median amount of BAF improved from 9.67 (range, $0-19$ ) at 1 month postoperatively to 12.38 (range, 3-20) at 12 months postoperatively. The magnitude of improvement significantly differed at these three follow-ups $(p=0.021)$. The amount of BAF in the IXT group at 1 month postoperatively was not significantly different from that of the controls $(p=0.533)$ and was better than that in the controls at 12 months postoperatively $(p=0.001)$.

\section{Accommodative Amplitude}

The patients' median accommodative amplitude in the right eye improved from $8.43 \pm 3.81$ at 1 month postoperatively to $8.64 \pm 3.67$ at 12 months postoperatively $(p=0.700)$, which was not significantly different from that of the controls $(7.37 \pm 2.16$, $p=0.689$ and $p=0.791$, respectively).

\section{Relationship Between Postoperative Binocular Functions and Postoperative Drift \\ Relationship Between Stereoacuity and Postoperative Drift}

Figures 2A,B show that the relationships between 1-month postoperative stereoacuity and postoperative drift for distance and near fixation were not significant $[r s=0.106(p=0.579)$ and $r s=0.143(p=0.450)$, respectively].

\section{Relationship Between the Fusional Vergence Amplitude and Postoperative Drift}

No significant correlations were found between the angle of postoperative drift and fusional vergence amplitudes at 1 month postoperatively for distance or near fixation in IXT patients (Figure 2).

\section{Classification of the IXT Group Into the Orthophoria/Heterophoria Group and Small Residual Manifest Angle Group}

By combining the measurements of the Worth 4-dot test and simultaneous prism and cover test (SPCT) at 1 month after surgery, the IXT group was divided into the orthophoria/heterophoria group (26 cases) and small residual manifest angle group (three cases with suppression and one case with diplopia). All four patients in the residual manifest group recovered normal single vision and motor function at the 12-month postoperative visit. Ocular alignment became better controlled to heterophoria at 12 months postoperatively. The other three cases in the orthophoria/heterophoria group exhibited reduced binocular vision functions and exodrifted significantly during the follow-up at 12 months postoperatively; thus, the operation was not successful in these patients. 


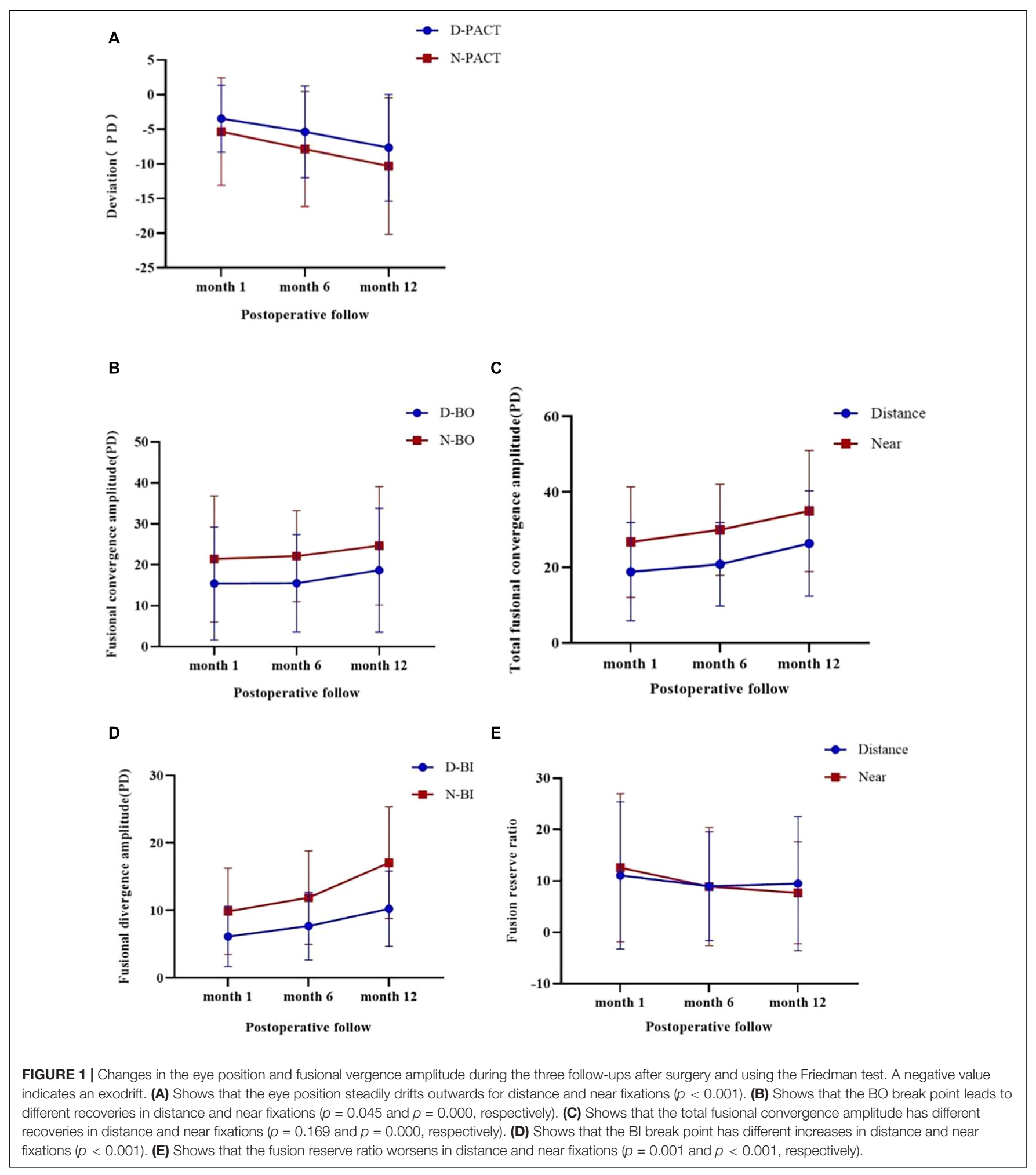

\section{DISCUSSION}

To our best knowledge, this is the first longitudinal study to evaluate the rehabilitation of binocular function in IXT after successful corrective surgery. Our results showed that most patients recovered binocular vision function after successful surgery, and the highest recovery rate was noted from postoperative months 1 to 6 . This rate improved steadily over the next 6 months. Although the patients recovered binocular functions after strabismus surgery, 

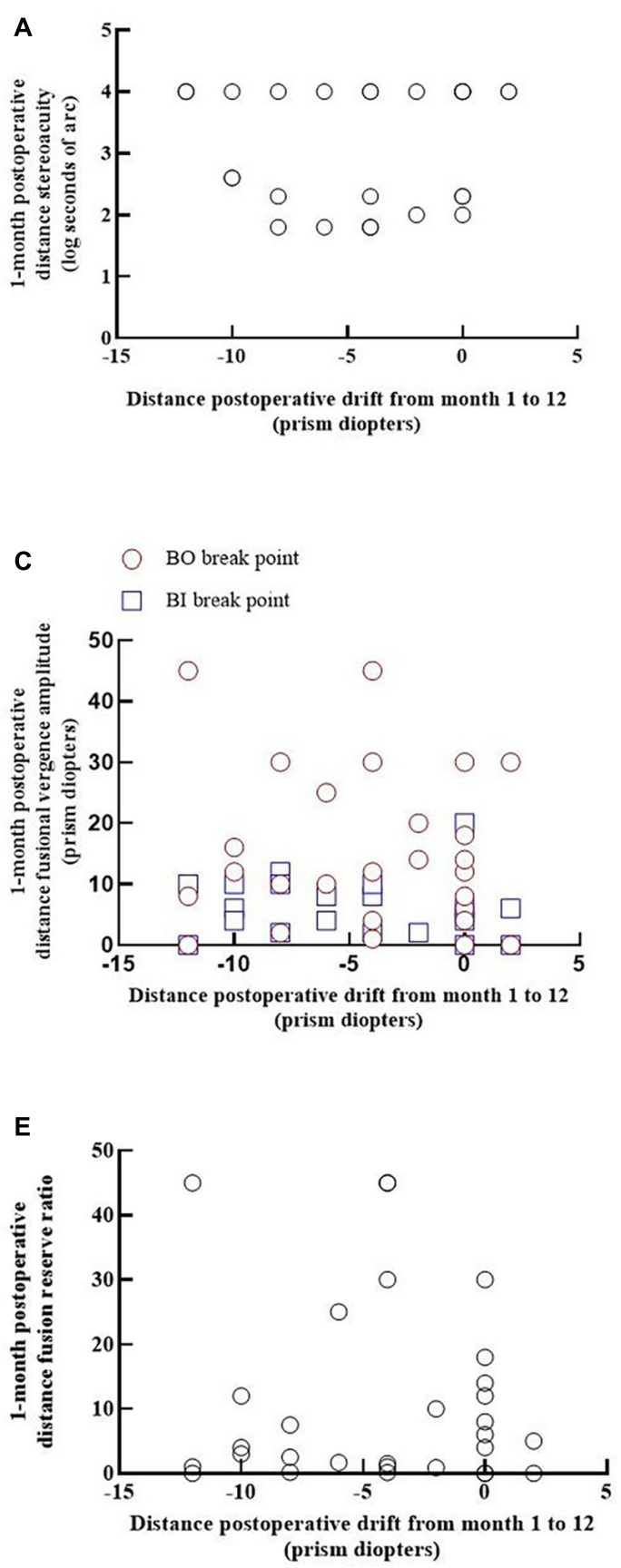
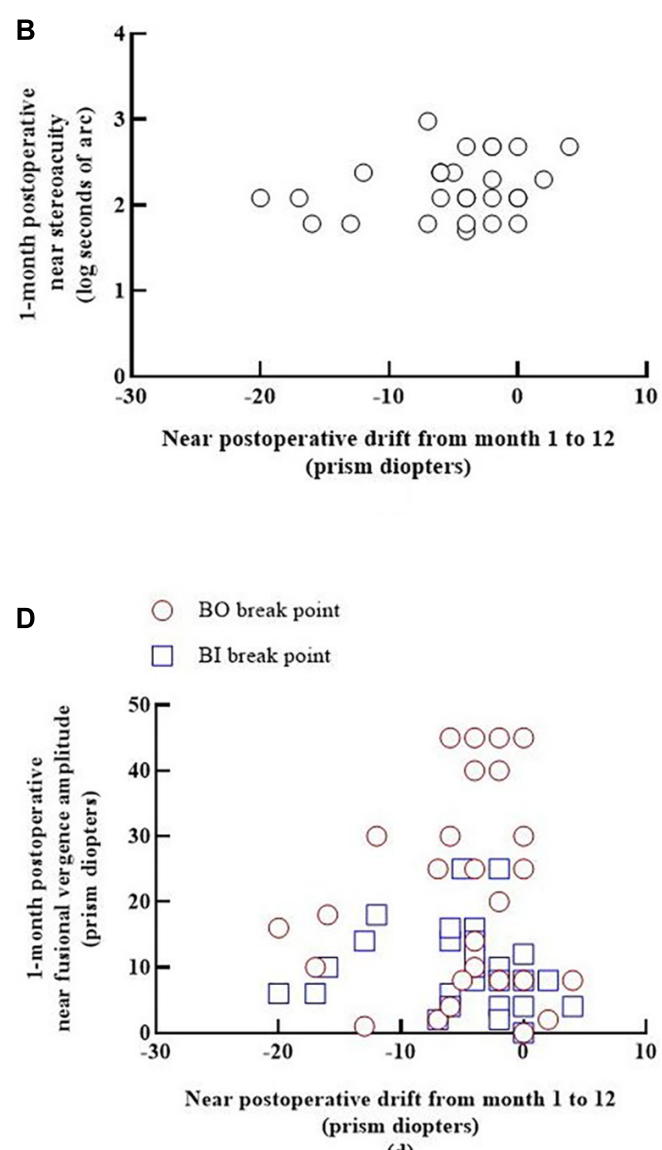

(d)

F

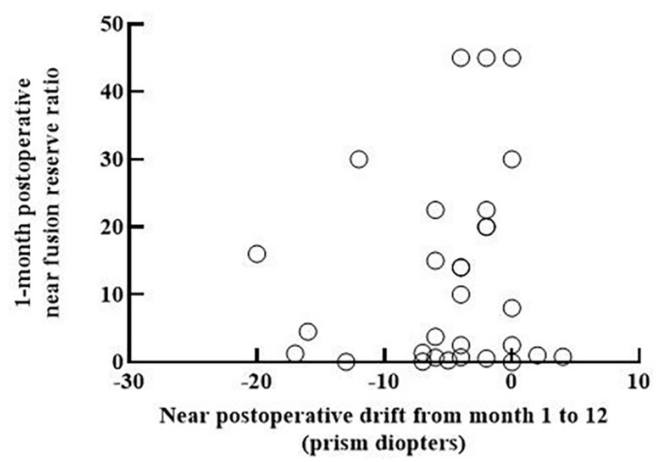

FIGURE 2 | Relationships between postoperative binocular functions and postoperative drift at distance and near fixations. Each panel shows the data from 30 patients. The positive values indicate esodrift, and the negative values indicate exodrift. (A,B) Show the relationship between 1-month postoperative stereoacuity and the postoperative drift from months 1 to 12 for distance and near fixations $[r$ s $=0.106(p=0.579)$ and $r S=0.143(p=0.450)$, respectively]. (C,D) Show the relationship between the convergence and divergence amplitude and postoperative drift at distance fixation (BO: $r s=-0.115, p=0.546 ; \mathrm{BI}: r s=-0.336, p=0.075)$ and at near fixation (BO: $r s=0.031, p=0.870$; $\mathrm{Bl}: r s=-0.268, p=0.159)$. (E,F) Show that the relationships between the 1-month postoperative fusion reserve ratio and postoperative drift from months 1 to 12 were not significant for distance or near fixation $[r s=0.003(p=0.987)$ and $r s=0.110(p=0.564)$, respectively].

some functions (i.e., near stereopsis, fusional convergence reserve, and the fusion reserve ratio) were still subnormal compared with those in controls. Few previous studies have evaluated the effects of postoperative binocular visual functions on the long-term stability of postoperative ocular alignment. Our study findings indicated no significant correlation between postoperative drift and postoperative binocular functions, suggesting that postoperative binocular functions may not be involved in the stability of postoperative ocular alignment. 
Stereoacuity is typically considered an important indicator of the severity of IXT and is used to determine surgical indications, particularly for distance stereopsis (Stathacopoulos et al., 1993; von Noorden and Campos, 2002; Holmes et al., 2007). The degree of recovery of stereopsis in IXT was inconsistent. O'Neal and associates (O'Neal et al., 1995) demonstrated that even with excellent postoperative alignment, the distance stereoacuity, particularly in the random dot test, does not recover fully. Notably, the age range of the subjects in that study was large, ranging from 5 to 87 years. Sharma et al. (2008) found that the mean distance stereoacuity of patients at 6 months postoperatively became similar to that of normal subjects. Our study also confirmed that distance stereopsis improved to the same level as that of the normal controls at the 6- or 12month follow-up. Adams et al. (2008) and Feng X. et al. (2015) found that near stereoacuity remained essentially unchanged following surgery. However, it should be noted that the subjects were assessed 6 weeks postoperatively and were not compared with controls. We found that near stereoacuity remained poorer in the patients at the last follow-up compared with that in the controls; however, it improved after surgery. Our results are consistent with those reported by Sharma et al. (2008), who found that even at 6 months postoperatively, the mean level of near stereopsis was still poor compared with that of control subjects.

The fusional vergence amplitude reflects a patient's ability to "control" a latent or intermittent deviation in IXT (von Noorden and Campos, 2002; Adams et al., 2008) or a patient's ability to maintain phoria in childhood X(T) (Wakayama et al., 2018). Kushner and Morton (1998) and Kushner (1999) found that exotropia becomes more pronounced when fusional convergence is suspended. Few studies have observed the postoperative fusional vergence amplitude and its longitudinal rehabilitation as well as its association with postoperative drift. The IXT group drifted outwards, and the exodeviation increased during the follow-up. As expected, the number of BI break points increased. We also found that the convergence reserve and total convergence amplitude improved in the follow-up period, but the values remained lower than those of the normal controls. This finding indicates that patients' fusional convergence ability may improve after the successful correction of IXT. Sharma et al. (2008) found no significant differences in the fusional vergence amplitude between patients at 6 months postoperatively and normal subjects. However, adult subjects were included in the study, and fusion data were collected after the angle of deviation had been corrected using prisms. Furthermore, Liebermann et al. (2012) believe that common measurement methods may not reveal the true divergence amplitudes. Most IXT patients have normal fusional divergence at near fixation, and approximately half show decreased fusional divergence at distance fixation. Hatt et al. (2011) found that the fusion reserve ratio correlates well with exodeviation control and may be useful in grading the severity of IXT. According to Sheard's criterion, the fusional convergence reserve should be twice the magnitude of the angle of deviation. Moreover, Yam et al. (2013) found that a fusional reserve ratio $\geq 2$ was an indicator of good control in patients. However, the postoperative fusional reserve ratio showed a decreasing trend and was not significantly correlated with postoperative drift in this study. The decreasing trend may be related to the change in exodrift after the surgery.

Consistent with most previous studies, we found that sensory fusion improved from before to after the operation (O'Neal et al., 1995; Yildirim et al., 1999; Feng X. et al., 2015; Wu Y. et al., 2020) and remained stable during the follow-up period without a trend of deterioration.

BAF improved at the last follow-up and exceeded that of the normal group, and the accommodative amplitude remained stable during the follow-up period and slightly exceeded that of the normal controls. Ha and his colleagues (Ha et al., 2016) suggested that the rehabilitation of accommodative loads at near fixation increases more in IXT patients than in normal controls, whereas binocular fusion remains consistent. One possible explanation is that disparity is a major driver of accommodation, and extra accommodation is a consequence in IXT patients.

According to Yildirim and her colleagues (Yildirim et al., 1999), better distance stereoacuity and central fusion before surgery are frequently associated with better surgical success in $\mathrm{X}(\mathrm{T})$. Beneish and Flanders (1994) found that overcorrection of poor preoperative stereopsis resulted in significant improvements in the surgical success rate and suggested that individuals with poor preoperative stereopsis may have good long-term alignment stability postoperatively. In this study, we found no significant relationship between postoperative stereopsis and postoperative drift. Furthermore, postoperative fusional vergence functions-i.e., fusional convergence reserve, total convergence amplitude, and fusional divergence amplitudewere not associated with postoperative drift and could not predict drift in the follow-up period. Considering the findings of previous studies (Hatt et al., 2008) and our study, other factors may exist in addition to postoperative binocular functions that are involved in postoperative alignment. For example, the surgery method and age at surgery will also affect the longterm stability of ocular alignment postoperatively. In this study, the operations were performed by the same doctor, the surgical procedures were all unilateral recession-resection procedures, and the school-aged children were older than seven years. The factors that interfere with postoperative eye position stability are relatively limited. Previous studies (Feng L. et al., 2015; Zhou et al., 2017; Wu H. et al., 2020) have shown that visual perception disorders occur in IXT patients; sensory imbalance persisted in surgically corrected subjects although they had normal levels of stereopsis (Park and Kim, 2013), and the level of sensory imbalance was subnormal in the follow-up period (Leow et al., 2010).

In the present study, we chose postoperative month 1 as baseline to observe the rehabilitation in binocular visual functions with IXT after surgery and excluded any immediate responses and complications related to surgery (e.g., pain, edema, bleeding, and reactions to anaesthetics). Furthermore, to minimize the influence of other factors, we set strict inclusion criteria (i.e., amblyopia, anisometropia, and vertical deviation). Similarly, we excluded patients with overcorrection (>5 PD) 
at 1 month postoperatively. Overcorrection is correlated with long-term motor benefits but has potential negative effects on binocular function (Leow et al., 2010; Choi et al., 2011; Park and Kim, 2013).

Our study has limitations. First, the control score plays a vital role in assessing the patients' ability to maintain phoria (Hatt and Gnanaraj, 2013; Wakayama et al., 2018). However, it is unsuitable for patients with IXT after surgery. Second, we included a limited number of patients, and the follow-up time was not sufficiently long. Binocular functions and eye position drift should be assessed in larger study populations and over longer periods. Binocular function measurements can be affected by the testing method-i.e., the fusional convergence reserve in IXT is greater when viewing a stereo target (Cooper, 1993) and reduced during intense light exposure (Campos and Cipolli, 1992). The testing conditions were uniform across all subjects in the present study; thus, there is likely no measurement bias in our results. Finally, we found a tendency of larger deviation before surgery with more drift after surgery, which would also affect the evaluation of the postoperative factors related to the postoperative alignment stability. A stricter definition of deviation before surgery and a larger sample would minimize the effect.

Binocular functions remained poorer in IXT patients at 1 month postoperatively than in the control group and continued to improve from 1 to 12 months. Some parameters of the binocular functions recovered to the same level as those in the normal controls, whereas some remained worse than those in the normal controls. No significant correlations were found between binocular functions after surgery and the stability of the alignment.

\section{REFERENCES}

Adams, W. E., Leske, D. A., Hatt, S. R., Mohney, B. G., Birch, E. E., Weakley, D. R. Jr., et al. (2008). Improvement in distance stereoacuity following surgery for intermittent exotropia. J. AAPOS 12, 141-144. doi: 10.1016/j.jaapos.2007.09. 015

Beneish, R., and Flanders, M. (1994). The role of stereopsis and early postoperative alignment in long-term surgical results of intermittent exotropia. Can. J. Ophthalmol. 29, 119-124.

Campos, E. C., and Cipolli, C. (1992). Binocularity and photophobia in intermittent exotropia. Percept. Mot. Skills 74, 1168-1170. doi: 10.2466/pms. 1992.74.3c.1168

Choi, J., Kim, S. J., and Yu, Y. S. (2011). Initial postoperative deviation as a predictor of long-term outcome after surgery for intermittent exotropia. J. AAPOS 15, 224-229. doi: 10.1016/j.jaapos.2010.12.019

Cooper, J. M. N. (1993). Intermittent exotropia, basic and divergence excess type. Binocul. Vis. Strabismus Q. 8, 187-216.

Donahue, S., Chandler, D., Holmes, J., Arthur, B. W., Paysse, E. A., Wallace, D. K., et al. (2019). A Randomized Trial Comparing Bilateral Lateral Rectus Recession versus Unilateral Recess and Resect for Basic-Type Intermittent Exotropia. Ophthalmology 126, 305-317. doi: 10.1016/j.ophtha.2018.08.034

Donahue, S. P. (2007). Clinical practice. Pediatric strabismus. N. Engl. J. Med. 356, 1040-1047.

Feng, L., Zhou, J., Chen, L., and Hess, R. F. (2015). Sensory eye balance in surgically corrected intermittent exotropes with normal stereopsis. Sci. Rep. 5:13075.

\section{DATA AVAILABILITY STATEMENT}

The original contributions presented in the study are included in the article/supplementary material, further inquiries can be directed to the corresponding author/s.

\section{ETHICS STATEMENT}

The studies involving human participants were reviewed and approved by the Ethics Committee of the Eye Hospital of Wenzhou Medical University. Written informed consent to participate in this study was provided by the participants' legal guardian/next of kin.

\section{AUTHOR CONTRIBUTIONS}

MX, FZ, JL, CW, and YW: technical assistance and guidance. JZ and SC: participating in the follow-up and contact patients to collect data. XY: research and academic guidance. All authors contributed to the article and approved the submitted version.

\section{FUNDING}

The study was supported by the National Natural Science Foundation of China grant NSFC (subject number: 82070995), Zhejiang Provincial Natural Science Foundation of China (subject number: LY19H120004), and the Medical Health Science and Technology Project of the Zhejiang Provincial Health Commission (subject number: 2019KY110).

Feng, X., Zhang, X., and Jia, Y. (2015). Improvement in fusion and stereopsis following surgery for intermittent exotropia. J. Pediatr. Ophthalmol. Strabismus 52, 52-57. doi: 10.3928/01913913-20141230-08

García, A., Cacho, P., Lara, F., and Megías, R. (2000). The relation between accommodative facility and general binocular dysfunction. Ophthalmic Physiol. Opt. 20, 98-104. doi: 10.1016/s0275-5408(99)00034-4

Ha, S. G., Jang, S. M., Cho, Y. A., Kim, S. H., Song, J. S., and Suh, Y. W. (2016). Clinical exhibition of increased accommodative loads for binocular fusion in patients with basic intermittent exotropia. BMC Ophthalmol. 16:77. doi: 10. 1186/s12886-016-0260-y

Hatt, S. R., and Gnanaraj, L. (2013). Interventions for intermittent exotropia. Cochrane Database Syst. Rev. 2013:CD003737.

Hatt, S. R., Leske, D. A., Mohney, B. G., Brodsky, M. C., and Holmes, J. M. (2011). Fusional convergence in childhood intermittent exotropia. Am. J. Ophthalmol. 152, 314-319. doi: 10.1016/j.ajo.2011.01.042

Hatt, S. R., Mohney, B. G., Leske, D. A., and Holmes, J. M. (2008). Variability of stereoacuity in intermittent exotropia. Am. J. Ophthalmol. 145, 556-561. doi: 10.1016/j.ajo.2007.10.028

Holmes, J. M., Birch, E. E., Leske, D. A., Fu, V. L., and Mohney, B. G. (2007). New tests of distance stereoacuity and their role in evaluating intermittent exotropia. Ophthalmology 114, 1215-1220. doi: 10.1016/j.ophtha.2006.06.066

Hutchinson, A. K. (2001). Intermittent exotropia. Ophthalmol. Clin. North Am. 14, 399-406.

Jie, Y., Xu, Z., He, Y., Wang, N., Wang, J., Lu, W., et al. (2010). A 4 year retrospective survey of strabismus surgery in Tongren Eye Centre Beijing. Ophthalmic Physiol. Opt. 30, 310-314. doi: 10.1111/j.1475-1313.2010.00716.x 
Kim, H. J., and Choi, D. G. (2016). Clinical analysis of childhood intermittent exotropia with surgical success at postoperative 2 years. Acta Ophthalmol. 94, e85-e89.

Kushner, B. J. (1999). Diagnosis and treatment of exotropia with a high accommodation convergence-accommodation ratio. Arch. Ophthalmol. 117, 221-224. doi: 10.1001/archopht.117.2.221

Kushner, B. J., and Morton, G. V. (1998). Distance/near differences in intermittent exotropia. Arch. Ophthalmol. 116, 478-486. doi: 10.1001/archopht.116.4.478

Lee, H. J., Kim, S. J., and Yu, Y. S. (2018). Long-term Outcomes After Same Amount of Bilateral Rectus Muscle Recession for Intermittent Exotropia With the Same Angle of Deviation. J. Pediatr. Ophthalmol. Strabismus 55, 319-325. doi: 10.3928/01913913-20180329-02

Leow, P. L., Ko, S. T., Wu, P. K., and Chan, C. W. (2010). Exotropic drift and ocular alignment after surgical correction for intermittent exotropia. J. Pediatr. Ophthalmol. Strabismus 47, 12-16. doi: 10.3928/01913913-20100106-04

Liebermann, L., Hatt, S. R., Leske, D. A., Yamada, T., Mohney, B. G., Brodsky, M. C., et al. (2012). Assessing divergence in children with intermittent exotropia. Strabismus 20, 11-16. doi: 10.3109/09273972.2012.655838

Mohan, K., and Sharma, S. K. (2020). Long-term Motor and Sensory Outcomes After Unilateral Lateral Rectus Recession-Medial Rectus Resection for Basic Intermittent Exotropia. J. Pediatr. Ophthalmol. Strabismus 57, 326-332. doi: 10.3928/01913913-20200731-01

O’Neal, T. D., Rosenbaum, A. L., and Stathacopoulos, R. A. (1995). Distance stereo acuity improvement in intermittent exotropic patients following strabismus surgery. J. Pediatr. Ophthalmol. Strabismus 32, 353-357. discussion 358, doi: 10.3928/0191-3913-19951101-06

Pan, C. W., Zhu, H., Yu, J. J., Ding, H., Bai, J., Chen, J., et al. (2016). Epidemiology of Intermittent Exotropia in Preschool Children in China. Optom. Vis. Sci. 93, 57-62. doi: 10.1097/opx.0000000000000754

Park, K. H., and Kim, S. Y. (2013). Clinical characteristics of patients that experience different rates of exodrift after strabismus surgery for intermittent exotropia and the effect of the rate of exodrift on final ocular alignment. J. AAPOS 17, 54-58. doi: 10.1016/j.jaapos.2012. 10.014

Pineles, S. L., Demer, J. L., Isenberg, S. J., Birch, E. E., and Velez, F. G. (2015). Improvement in binocular summation after strabismus surgery. JAMA Ophthalmol. 133, 326-332. doi: 10.1001/jamaophthalmol.2014.5265

Pineles, S. L., Ela-Dalman, N., Zvansky, A. G., Yu, F., and Rosenbaum, A. L. (2010). Long-term results of the surgical management of intermittent exotropia. J. AAPOS 14, 298-304. doi: 10.1016/j.jaapos.2010.06.007

Repka, M. X., Chandler, D. L., Holmes, J. M., Donahue, S. P., Hoover, D. L., Mohney, B. G., et al. (2020). The Relationship of Age and Other Baseline Factors to Outcome of Initial Surgery for Intermittent Exotropia. Am. J. Ophthalmol. 212, 153-161. doi: 10.1016/j.ajo.2019.12.008

Sharma, P., Saxena, R., Narvekar, M., Gadia, R., and Menon, V. (2008). Evaluation of distance and near stereoacuity and fusional vergence in intermittent exotropia. Indian J. Ophthalmol. 56, 121-125. doi: 10.4103/0301-4738.39116

Stathacopoulos, R. A., Rosenbaum, A. L., Zanoni, D., Stager, D. R., McCall, L. C., Ziffer, A. J., et al. (1993). Distance stereoacuity. Assessing control in intermittent exotropia. Ophthalmology 100, 495-500. von Noorden, G. K., and Campos, E. C. (2002). Binocular Vision and Ocular Motility: Theory and Management of Strabismus. Vol 6th ed. Missouri: CV Mosby.

Wakayama, A., Seki, Y., Takahashi, R., Umebara, I., Tanabe, F., Abe, K., et al. (2018). Role of fusional convergence amplitude in postoperative phoria maintenance in children with intermittent exotropia. Jpn. J. Ophthalmol. 62, 307-314. doi: 10.1007/s10384-018-0585-6

Wang, J., Hatt, S. R., O’Connor, A. R., Drover, J. R., Adams, R., Birch, E. E., et al. (2010). Final version of the Distance Randot Stereotest: normative data, reliability, and validity. J. AAPOS 14, 142-146. doi: 10.1016/j.jaapos.2009.12. 159

Wick, B., Yothers, T. L., Jiang, B. C., and Morse, S. E. (2002). Clinical testing of accommodative facility: part 1. A critical appraisal of the literature. Optometry $73,11-23$.

Wu, H., Li, X., Tang, Y., Xu, Q., Zhang, X., Zhou, L., et al. (2020). Optimal Stereoacuity Reveals More Than Critical Time in Patients With Intermittent Exotropia. Front. Neurosci. 14:133. doi: 10.3389/fnins.2020.00133

Wu, H., Sun, J., Xia, X., Xu, L., and Xu, X. (2006). Binocular status after surgery for constant and intermittent exotropia. Am. J. Ophthalmol. 142, 822-826. doi: 10.1016/j.ajo.2006.06.045

Wu, Y., Xu, M., Zhang, J., Zhou, J., Wan, M., Dai, Z., et al. (2020). Can Clinical Measures of Postoperative Binocular Function Predict the Long-Term Stability of Postoperative Alignment in Intermittent Exotropia? J. Ophthalmol. 2020:7392165.

Yam, J. C., Chong, G. S., Wu, P. K., Wong, U. S., Chan, C. W., and Ko, S. T. (2013). A prospective study of fusional convergence parameters in Chinese patients with intermittent exotropia. J. AAPOS 17, 347-351. doi: 10.1016/j.jaapos.2013. 03.023

Yildirim, C., Mutlu, F. M., Chen, Y., and Altinsoy, H. I. (1999). Assessment of central and peripheral fusion and near and distance stereoacuity in intermittent exotropic patients before and after strabismus surgery. Am. J. Ophthalmol. 128, $222-230$.

Yu, X., Ji, Z., Yu, H., Xu, M., and Xu, J. (2016). Exotropia Is the Main Pattern of Childhood Strabismus Surgery in the South of China: a Six-Year Clinical Review. J. Ophthalmol. 2016:1489537.

Zhou, J., Wang, Y., Feng, L., Wang, J., and Hess, R. F. (2017). Straightening the Eyes Doesn't Rebalance the Brain. Front. Hum. Neurosci. 11:453. doi: 10.3389/ fnhum.2017.00453

Conflict of Interest: The authors declare that the research was conducted in the absence of any commercial or financial relationships that could be construed as a potential conflict of interest.

Copyright (c) 2021 Peng, Xu, Zheng, Zhang, Chen, Lou, Wang, Wang and Yu. This is an open-access article distributed under the terms of the Creative Commons Attribution License (CC BY). The use, distribution or reproduction in other forums is permitted, provided the original author(s) and the copyright owner(s) are credited and that the original publication in this journal is cited, in accordance with accepted academic practice. No use, distribution or reproduction is permitted which does not comply with these terms. 